\title{
A meeting of minds: interdisciplinary research in the health sciences in Canada
}

\author{
Judith G. Hall, Lesley Bainbridge, Alison Buchan, Alastair Cribb, Jane Drummond, Carlton Gyles, \\ T. Philip Hicks, Carol McWilliam, Barbara Paterson, Pamela A. Ratner, Elizabeth Skarakis-Doyle, \\ Patty Solomon
}

$\infty$

See related article page $76 \mathrm{I}$

\section{ABSTRACT}

Brought together by the newly formed Canadian Academy of Health Sciences (CAHS), recognized national leaders in the 6 health sciences disciplines consider the environment for conducting interdisciplinary health research (IDHR) in Canada. Based on first-hand knowledge and thoughtful reflection, the authors argue that although much progress has been made in support of IDHR in Canada, the practical experience of researchers does not always bear this out. This article examines government, industry and academia to identify the cultural and structural characteristics that demand, promote or prevent IDHR in each sector. At its heart is the question, How can universities best support and enhance IDHR, not only for the benefit of science, but also to meet the growing needs of industry and government for intellectual capital?

Focusing on the predominant health sciences disciplines, the authors define IDHR as a team of researchers, solidly grounded in their respective disciplines, who come together around an important and challenging health issue, the research question for which is determined by a shared understanding in an interactive and iterative process. In addition, they suggest that IDHR is directly linked to translational research, which is the application of basic science to clinical practice and the generation of scientific questions through clinical observation.

This analysis of academic, industry and government sectors is not intended to offer rigorous data on the current state of IDHR in Canada. Rather, the goal is to stimulate researchpolicy dialogue by suggesting a number of immediate measures that can help promote IDHR in Canada.

Recommended measures to support IDHR are aimed at better resourcing and recognition (by universities and granting agencies), along with novel approaches to training, such as government- and industry-based studentships. In addition, we recommend that professional organizations reconsider their policies on publication and governance. Although intended to maintain professional scopes of practice, these policies also serve to entrench disciplinary boundaries in research.
We conclude by suggesting a number of research questions for a more rigorous assessment of the climate for IDHR in Canada. We call for an inventory and comparative analysis of academic centres, institutes and consortiums in Canada that strive to facilitate IDHR; an examination of the impact of professional organizations on health research, and on IDHR in particular; and a systematic review of research training opportunities that promote IDHR, with a view to identifying and replicating proven models.

CMAJ 2006;175(7):763-71

We are not students of some subject matter, but students of problems. And problems may cut right across the borders of any subject matter or discipline. ${ }^{1}$ - Sir Karl R. Popper, philosopher of science

W hat do avian influenza, obesity, breast cancer and SARS have in common? They are all major and complex Canadian public-health issues that require significant effort on the part of multiple health-science disciplines. Their characteristics and contributing factors are not bound by any disciplinary borders. In the face of these issues and others like them, health researchers and practitioners alike can and must draw on expertise and experience from across health science disciplines, and even disciplines based outside of health.

As obvious as this seems, the mechanics and practicalities of an interdisciplinary approach are rarely compatible with the traditional structures of scientific enquiry that still reign within our universities, academic health care settings and research agencies at all levels. There has been much focus in recent years on developing an interdisciplinary research environment, most notably through the advent of the Canadian Institutes of Health Research (CIHR). Yet, in most academic contexts, the fundamental building blocks of the research establishment training, funding, administration of grants, peer review, publishing and professional recognition - have been slow to shift to include interdisciplinary enquiry, despite the innovative and laudable architecture of research that has been the very foundation of the CIHR since its inception in 2000. 
This article is the product of multiple intensive roundtable discussions among national leaders of the 6 health sciences disciplines during the fall of 2004 and spring of 2005 (unpublished report, $2005^{\star}$ ). Brought together by the newly formed Canadian Academy of Health Sciences (CAHS) to develop a framework for assessing Canadian interdisciplinary health research (IDHR) that would stimulate policy dialogue, the authors were selected because of their outstanding research credentials and experience in IDHR. The topic was chosen specifically to explore the potential for collaboration between the 6 disciplines and to highlight Canada's unique opportunities for this kind of research. In addition to representing the 6 health-science disciplines, the authors are broadly based geographically, represent a range of ages and are well-balanced by sex.

In what follows, we argue that although much progress has been made in support of IDHR in Canada, the practical experience of researchers does not always bear that out. We examine 3 key sectors for IDHR in Canada - government, industry and academia - to identify the cultural and structural characteristics that demand, promote or prevent IDHR in each. From a perspective of academic research, we ask: How can universities best support and enhance IDHR, not only for the benefit of science, but also to meet the growing human resource and expertise needs of government and industry? The analysis presented here is drawn from our own experiences combined with focused, thoughtful reflection. It is not intended to offer rigorous data on the current state of IDHR in Canada. Rather, we conclude by proposing a number of possible paths that such an analysis might take.

Although there has been a great deal of discussion in recent academic and education literature on interprofessional education and practice, these types of collaboration are beyond the scope of this article. In the United States, the Institute of Medicine recently published a very thoughtful book about interdisciplinary work in science in general. ${ }^{2} \mathrm{We}$ focus instead on the research endeavour, and specifically on interdisciplinary research in the health sciences (i.e., IDHR). For the purposes of this discussion, we understand interdisciplinary research to mean collaboration between the predominant health sciences disciplines; however, it need not be limited to these. Indeed, other disciplines such as psychology, epidemiology, ethics and other social and "bench" sciences may well be considered for fruitful collaboration. In addition, the fields of law, business, education, economics and political science undoubtedly have much to offer interdisciplinary research in the health sciences in Canada.

\section{Definitions}

The problem of defining interdisciplinary research is the first step in understanding how best to turn it into common practice within the academic health sciences. The literature offers several terms that are often, erroneously, used interchange-

* Readers may contact the corresponding author of this article for a copy of the unpublished report referred to in the text. ably: interdisciplinary, multidisciplinary, interprofessional, cross-disciplinary and transdisciplinary.

Marts $^{3}$ referred to an Institute of Medicine (IOM) report when she described interdisciplinary research as "a cooperative effort by a team of investigators, each expert in the use of different methods and concepts, who have joined in an organized program to attack a challenging problem" (p. 502). Transdisciplinary was referred to in the IOM report as "the development of a common conceptual framework that bridges the relevant disciplines and that can serve as the basis for generating new research questions directly related to the defined problems" (p. 503). ${ }^{3}$ Stokols and colleagues ${ }^{4}$ used Rosenfield' ${ }^{5}$ work to justify the identification of the term transdisciplinary as a process that involves shared concepts, as well as integration and extension of discipline-specific theories, to address common research issues. Stokols and colleagues ${ }^{4}$ have contrasted this with multidisciplinary research, in which independent or sequential research is said to be focused on a common problem, and interdisciplinary research, in which greater sharing occurs among participants anchored in their respective disciplinary perspectives but which, in the view of Stokols' group, “... stops short of achieving novel and integrative conceptual models" (p. 204).

We agree with Stokols and colleagues that the complexity of collaborative activity requires a high degree of confidence in disciplinary knowledge and practice; nevertheless, we argue that integration is, in fact, part of the key to this type of work. By its very essence, interdisciplinary scholarship requires the deconstruction of knowledge and identity, which is then reconfigured into new forms of knowledge and action. Researchers working in interdisciplinary realms must demonstrate the ability to move between interdisciplinary and disciplinary scholarship. This ensures that they do not lose sight of the disciplinary strengths they bring to their interdisciplinary work.

Of course these definitions may be quite different in the current Canadian context of health sciences research, as evidenced by the structure of CIHR strategic grants, existing university-based research centres and the research practices commonly found in industry and government. We recognize that defining IDHR for Canadians will require ongoing discussion, and suggest the following thoughts to start: IDHR may be seen as "a team of researchers, solidly grounded in their respective disciplines, that come together around an important and challenging health issue, the research question for which is determined by a shared understanding in an interactive and iterative process" (unpublished report, 2005*).

In addition, we suggest that, by its very nature, IDHR is directly linked to "translational" research, or what the Association of Professors of Medicine has defined as "... the application of basic scientific discoveries into clinically germane findings, and simultaneously, the generation of scientific questions based on clinical observations."

\section{Promoting interdisciplinary health research}

The issue of how to promote IDHR on campuses across Canada is not new. In fact, the growing interdisciplinary 
commitment of government-supported research funding institutions in Canada is readily apparent, most notably through the mandate of the CIHR. Created in 2000 to replace the Medical Research Council (MRC) of Canada and subsequently expanded to accommodate the National Health Research and Development Program (NHRDP) transferred to it by Health Canada, CIHR is a testament to this direction. ${ }^{7}$ In the few short years since its inception, CIHR has created synergy by combining the biomedical focus of the MRC with the health systems and population health focus of NHRDP. As then-Health Minister Allan Rock stated, ${ }^{8}$

The CIHR will help to integrate health-research activity by fostering linkages and breaking down barriers that have existed among the different fields of health research: biomedical research, clinical research, research respecting health systems, health services, the health of populations, societal and cultural dimensions of health and environmental influences on health. This new approach will create a vibrant environment that will recognize the importance of collaborative research for improving the health and well-being of Canadians and for building a high-quality health system.

Through its way of functioning, CIHR has perhaps achieved its greatest contribution to the promotion of interdisciplinary research in the health sciences. Structurally, CIHR demands interdisciplinary collaboration with its mandate of taking research "from bench to bedside." In so doing, the organization crosses 4 pillars (biomedical, clinical, health systems and services, and population and public health) and offers 13 research themes: aboriginal peoples' health; cancer research; circulatory and respiratory research; gender and health; genetics; health services and policy research; aging; human development and child and youth health; infection and immunity; musculoskeletal health and arthritis; neurosciences, mental health and addiction; nutrition, metabolism and diabetes; and population and public health. In addition to encouraging and supporting individual IDHR projects that link the disciplines within biomedical science, medicine and other health science disciplines, CIHR has actively advanced the IDHR agenda by designating funds for cutting-edge initiatives such as the Strategic Training grants for interdisciplinary training programs, the New Emerging Team grants and the Interdisciplinary Capacity Enhancement Grants.

With $30 \%$ of its research funding reserved for strategic initiatives and $70 \%$ for investigator-driven research, between the fiscal years of 1999/2000 and 2003/04, CIHR achieved a considerable shift toward translational research and the funding of researchers other than bench scientists and physicians, thereby promoting IDHR. Funding over the same period for population-health and health-services research grew proportionally more quickly (by about $18 \%$ and $45 \%$, respectively) than that for clinical $(\sim 5 \%)$ and biomedical re$\operatorname{search}(\sim 4 \%)$.

In addition, one of the most important innovations of CIHR increasingly has been to promote and foster strategic partnerships with government and the private sector. These unprecedented partnerships, which were unheard of in the antecedent Medical Research Council, testify to CIHR's foresight and innovation. Indeed, overlap is inevitable between CIHR's academic projects and areas of interest and the activities of researchers in government ministries such as Health Canada, Indian and Northern Affairs, Industry Canada and a multitude of other science-based departments and agencies.

The innovative funding structure of CIHR is complemented by provincial funding bodies such as the Michael Smith Foundation for Health Research (MSFHR) in British Columbia, the Alberta Heritage Foundation for Medical Research and the Fonds de recherche en santé du Québec (FRSQ). These organizations promote research collaborations through their innovatively structured grants. The MSFHR, for instance, has recently launched 8 population-based health research networks focusing on priority populations in BC, each with a mandate to build critical mass through the development of provincial, national and international linkages among researchers and policy makers.

Driven by complex health issues, the structure of these networks is echoed in industry. Here too, the clear trend is toward IDHR networks that transcend organizational barriers, with industry, government and university scientists involved in joint ventures that target specific problems. Canada's Networks of Centres of Excellence and organizations such as Genome Canada are just 2 examples. Such endeavours typically target translational research and often include support from not-for-profit corporations.

These efforts are aided by government incentives that provide tax credits for research and development, as well as by technology such as the Canadian Network for the Advancement of Research, Industry and Education (CANARIE), which facilitates transfer of information. Government support of infrastructure and personnel through the Canada Foundation for Innovation (CFI) also facilitates IDHR. These interdisciplinary and intersectoral collaborations arise from a risksharing perspective. They allow the participants to explore areas that they might not pursue otherwise.

For all of the innovation and active promotion of novel collaborations at the macro level, however, it can hardly be said that IDHR has been normalized on the campuses of Canadian universities and other academic medical institutions. Nor can it be said that academia is keeping pace with the management and administrative procedures required to meet the interdisciplinary knowledge needs of government and industry. Yet Canada remains a highly respected leader in health research and a prized destination for many world-class scientists. Information technology that links researchers and databases across the country, combined with a socialized medical system and an enlightened research policy and funding environment, set Canada apart. The challenge now is to maintain and enhance this global position by reflecting on some critical questions.

How can the Canadian academic health-research establishment adapt, not only to seize interdisciplinary opportunities to advance science, but also to provide the intellectual capital so sought by industry and government? In short, how can universities train, nurture and promote a new kind of re- 
searcher whose skills and competencies are fully recognized in all research sectors?

\section{What should universities know about the research culture of government and industry?}

In recent years, Canada has experienced an explosion of growth and opportunity in the life-sciences sector of the economy. The major universities, through university-industry liaison offices (UILOs), have been active players in this expansion, fostering and promoting spin-off companies and taking a leadership role in ensuring intellectual-property protection for promising new products and technologies.

In a similar way, by promoting translational research that has direct application to either product or policy development, universities are building important links to government. Academic researchers who devote some of their time to national councils, governing bodies, expert panels and the like are active translators of research in the service of the public. Despite their efforts to build relationships with both the public and the private sectors, however, how well do universities actually train researchers to work with (or in) these contexts? Do graduates adequately understand the motivations, demands, constraints and customs of government or industry? Are they confident in a team research environment and conversant with multiple approaches to a research question? Can they perform under rigorous time constraints and maintain communication with team members and stakeholders? Equipping graduates with the skills and competencies of IDHR would go a long way toward helping prepare them for the "culture shock" of industrial and government research collaborations.

More than their university counterparts, researchers based in governments and industry are bound by the concept of return on investment. Public- and private-sector researchers are compelled to observe strict milestones and work to dictated timelines. In addition, the goal-oriented research of these sectors requires clear tasks and expected outcomes. In contrast, university researchers respond to various criteria not often faced in industry or government, such as teaching responsibilities, graduate student supervision, thesis committee membership and the academic code of "publish or perish."

For industry-based research, profit is the primary goal. Consequently, projects tend to be tightly focused and highly efficient. A top-down approach allows managers to assemble teams of consulting researchers for single projects with clearly defined outcomes. Not bound by any disciplinary tradition, industry benefits from the flexibility to recruit whoever might be needed to get the job done.

Unlike industry, government has a mandate for public service. Canada's Science and Technology Partnerships program (www.infoexport.gc.ca/science/partnerships-en.htm) identifies 4 roles for government in science and technology: funder, facilitator, performer and regulator. Because of these roles, government departments are often cautious about the possibilities of perceived or real conflicts of interest between the performance or application of research and its regulation. For instance, it may be difficult for a government department to obtain information or samples from individuals or private organizations that fear potential repercussions arising from a regulatory role related to findings by the department during the conduct of the research.

In government, the motivations, questions and operating procedures of its research are often very different from those in industry. Researchers must demonstrate that their topic is of significant public concern and must be diligently transparent in all research activities. Like those in industry, however, government laboratories tend not to be organized according to strict scientific disciplines and are poised to undertake interdisciplinary research by themselves or in collaboration with universities and/or industry. The values of interdisciplinary research and of teamwork are emphasized and researchers often work in teams assembled to tackle complex problems. There is an emphasis on short-term objectives, but the expected result is not always a product or service. In some projects, the goal directly addresses a concern in the health care system; in others, the goal is to provide information that will eventually contribute to policy development.

There are many cooperative research activities that involve both industry and government; several include universities, as well. In these cases the interests of the various groups are usually shaped by the specific requirements of a funding organization, but the cultural differences between the sectors are likely to persist.

Interestingly, as the frequency and scope of collaborations among industry, government and universities increase, we are seeing a merging of cultures in interdisciplinary research in all areas, including the health sciences. That said, there still is a paucity of literature on the processes, values, interests and culture that influence interdisciplinary research in industry and government in Canada and other countries. As Canada positions itself to attract international research funds, an understanding of the tax and regulatory environment, as well as the advantages of the Canadian health care system for IDHR, need also to be defined in order to be globally competitive.

\section{Does academic culture help or hinder interdisciplinary possibilities?}

The outcome-oriented, time-constrained and multi-investor, multiple-stakeholder research that is characteristic of the government and industrial sectors appears not to share a fundamental motivator with the academic sector; that is, the understanding that research is essentially knowledge production, systematically pursued, which advances science best when solutions to earlier questions lead to additional questions. To many university-based practitioners, research is an inherently iterative and evolutionary process that is generally not specifically directed toward eventual goals or outcomes (although the need to publish and gain recognition for career advancement may be considered a goal).

Indeed, although goal-oriented research lends itself easily 
to interdisciplinary solutions, postsecondary academic institutions are not traditionally configured to approach research in this way. ${ }^{9}$ So, are they equipped to produce researchers who are able to take advantage of innovative solutions and perform in a variety of research and knowledge-translation contexts? Through innovative structures such as research centres and institutes, and through mechanisms such as faculty cross-appointments and joint degrees, Canadian universities and provincial and federal funding agencies have made a great deal of progress in facilitating IDHR. Indeed, the ability to compare and test these models across provinces is one of Canada's great advantages. However, just as Birnbaum argued 25 years ago,${ }^{10}$ the discipline-bound structure of most Canadian universities implies that what is valued within academic culture is the independent scholar from a singular discipline. Today, the persistence of long-held academic traditions and administrative processes make sustainable interdisciplinary training and research a still-distant goal.

Historically, the challenges are based in numerous structural, cultural and organizational features of the university environment. As the primary centre for knowledge creation, it is organized physically and conceptually around specific knowledge content areas such as the sciences and humanities. Consequently, the medical sciences departments of biochemistry and anatomy are housed in buildings often at one end of campus, while social scientists (e.g., psychologists and sociologists) and ethicists (from the philosophy department) are located at another. Even university libraries are arranged to support distinct disciplinary identities. Within each separate and often isolated location, a distinctive culture flourishes, steeped in its own symbolic generalizations, language, models, principles and, thus, pathways to knowledge creation. ${ }^{11}$

These foundational elements allow for cohesion within a discipline via a common language, the identification and framing of research questions, and the establishment of standards for evidence..$^{12}$ In this way, the identity of the discipline is formed and maintained. Other academic organizational structures reinforce this identity through control of resource allocation (e.g., faculty positions, budgets, space allocation), curricula and criteria for granting degrees.

Players outside academia also contribute to the entrenchment in the research establishment of disciplinary identity. Among these players are the granting agencies that set and fund the national research agenda, which maintain boundaries by constraining the selection process within tightly defined review panels. Other external influences are the available venues for publication of research results, where editorial boards consist of discipline-bound experts and members of professional societies. As Giacomini ${ }^{11}$ stated, "Disciplinary cultures shape members' identities, relationships and even the knowledge that is created by determining what counts as work and even whose work counts."

In the academic health sciences, disciplinary identity is further cemented (not to mention, complicated) by the multiple roles of participants as educators, researchers and professional practitioners, who are governed by diverse professional bodies. Health sciences departments in Universities often serve 3 masters: the university, with its academic standards and cultural values; the professional colleges that accredit academic programs, and the associations that govern continuing education and recognize research according to their standards and values; and the hospitals and other health care institutions, where separate departments, programs and hierarchies are entrenched. Although it is hoped that these 3 streams coincide, they are not always complementary and in fact may compete and thereby undermine any attempts at interdisciplinary collaboration. This can happen when professional scopes of practice are confused with knowledge-based disciplinary boundaries, or when the hierarchy of the health care team is imposed on the research team. The organizational structures inherent in a university may create challenges for IDHR; so, too, may the policies

\section{A summary of potential measures to support IDHR}

\section{Resource provision}

Academic institutions

- Make workload adjustments to allow time for IDHR

- Provide physical space for meetings and collaboration

- Provide administrative support: coordinators, librarians

Funding agencies

- Enhance innovative RFPs and seed money

- Collaborate with government and industry on studentships

- With universities, devise ways to credit non-investigatorinitiated research

\section{Recognition and reward}

- Include members with IDHR experience on peer-review, hiring and promotion committees

- Candidates to include statement of contribution to collaborative work and letters of support from coinvestigators or PDF supervisor

- Consider the number of publications in journals from more than 1 discipline, and develop other quantifiable criteria

- Support the Common CV Network process by NAPHRO

- Incentives: merit-based awards for IDHR

Training

- Consider the contribution of interprofessional education to IDHR

- From undergraduate to postdoctoral levels, promote problem-based methods

- Develop interdisciplinary curriculum in collaboration with funding agencies

- Strengthen novel training schemes: clinician-scientist and co-op studentships in government or industry

\section{Professional organizations}

- Promote IDHR among the professions

- Reconsider and amend curriculum, governance and licensing structures that entrench boundaries

- Revisit and adapt policies that discourage publication in journals outside the "home" discipline

Note: IDHR = interdisciplinary health research, RFPs $=$ requests for proposals, PDF $=$ Postdoctoral Fellowship, $\mathrm{CV}=$ curriculum vitae, NAPHRO = National Alliance of Provincial Health Research Organizations. 
and traditions of professional associations and health care delivery systems.

This isolating effect of the professions can also be felt even when efforts are made to support interdisciplinary work. Although many Canadian universities now structure faculties of health sciences to facilitate collaboration, some of these structures still reinforce chasms such as those between medicine and the other allied health sciences. Often, only an illusion of IDHR is created by collaboration among physicians from different medical specialties. Unfortunately, the broader determinants of health and translational research are typically overlooked within these limited efforts. In addition, the university structure, which ties together various health science disciplines, is often understood to exist simply for administrative efficiency or to avoid the potential for power hierarchies to develop. Whether real or perceived, such motives serve only to create cynicism, which may impede, rather than promote, IDHR.

The academic career ladder is a feature that also serves to maintain disciplinary boundaries. Those engaged in IDHR can be disadvantaged with respect to recruitment into faculty positions. IDHR candidates may have fewer publications in journals with high impact factors, which tend to be discipline-based, or may publish less frequently simply because collaborative work takes longer. ${ }^{13}$ In addition, recruitment, promotion and peer-review committees may also have difficulty evaluating a candidate's contributions to projects and articles - admittedly a problem associated with multiauthored publications in general, not just interdisciplinary publications. (We note that steps are being taken to address this. For instance, the Natural Sciences and Engineering Research Council of Canada requires grant applicants to identify their contributions to multiauthored work.) The problem of disciplinary boundaries is exacerbated by the common practice of housing interdisciplinary research in (often physically separate) centres and institutes within Faculties of Graduate Studies, where individual researchers must identify a departmental sponsor. The department itself may stand to benefit very little from the candidate's training or research activities, which are focused within the interdisciplinary centre. Therefore, there may be little incentive for hiring such an individual.

Similarly, the process of tenure within academic culture does not lend itself easily to an interdisciplinary environment. Traditional metrics for tenure require a person to hold considerable peer-reviewed grant funding in his or her name; publish in relevant prestigious discipline journals; to contribute to professional training and academic teaching in a particular discipline; and to have an international reputation within their discipline. The challenge of demonstrating one's contribution to multi-investigator grants and multiauthored publications is obvious. In addition, the competitive nature of the university environment often requires researchers to justify their space and compete for recognition and promotion. ${ }^{14}$ This is incompatible with IDHR, which requires not only the physical space for research meetings and collaborative discussion but also intellectual space for rich intercourse across disciplines.
Outside the university itself, the structures that constitute the research establishment (among them granting agencies, publications and professional organizations) tend to reinforce boundaries that resonate more with traditional university-based research than with research in the other sectors, namely, government and industry. For instance, research granting agencies that insist on a single principal investigator can unintentionally undermine IDHR researchers' commitment to collaboration. Similarly, most refereed journals require that authors be listed in order of decreasing contribution to the endeavour; however, IDHR teams produce manuscripts that are evidence of their unified work. The ordering of authorship contravenes the tenets of interdisciplinarity. ${ }^{14}$ Additionally, recognition within professional societies requires that members publish in journals within their discipline, which further inhibits collaboration.

With the usual criteria for funding, publishing, appointment, tenure and promotion based on the notion of individual scholarship, it is unsurprising that it is more often the well-established senior researchers who are likely to explore new directions across disciplines. ${ }^{15}$ Ironically, their new interests may come at a time in their careers when work habits are also well established. The interpersonal practices, common language and shared vision required for IDHR may therefore be more difficult for such researchers to adopt. Ideally, these competencies would be instilled very early in a graduate student's career, not only serving IDHR but also leading eventually to career options in other research sectors.

This said, the current culture and practices of the university can influence students' ability and willingness to engage in IDHR, which are often relatively invisible on campus. Students are generally admitted to programs within a single discipline; the opportunity to work as a team member in an IDHR project most often occurs by happenstance. ${ }^{16}$ The need to identify research mentors with complementary strengths and interests is often a challenge for students. Instead, the more common approach is that a supervisor's research generally provides the foundation for the student's graduate work, consequently entrenching students in their supervisors' discipline and further reinforcing boundaries. ${ }^{17}$ Furthermore, students must first consider whether the inclusion of IDHR in a graduate thesis is even acceptable to the university or to the discipline. ${ }^{17}$

Although the format for graduate research training has remained largely traditional, its potential to effect the cultural shift toward IDHR is very great. New researchers with a solid disciplinary foundation and the skills and competencies to engage beyond their discipline represent the vanguard in health sciences research and form the cadre that will be best able to serve science, industry and the public. Research training therefore offers important opportunities to engage the "best and brightest," who might not otherwise find the right fit for their research interests.

In the academic health sciences, the explosion of interest in and recent federal commitments to interprofessional education raise the question of whether such collaboration can also inform future directions for interdisciplinary research 
and research training. In systematic reviews, $\operatorname{Cooper}^{18}$ and Freeth $^{19}$ and their respective coauthors have shown that interprofessional learning resulted in positive changes in knowledge, skills, attitudes and beliefs of the students; however, no effects were noted on professional practice behaviour, nor was any mention made of the effects of promotion of collaborative research. Although only a small percentage of health professional students will pursue research careers, interprofessional training seems a promising direction to pursue, given that it likely promotes the collaborative competencies essential for interdisciplinary research as well.

Another promising trend in training is support for clinician-scientists: "researchers who have a health-science professional degree along with research training at the master's or doctoral level, and who perform health-science research of any type as a core professional activity." Clinicianscientists face unique challenges such as poor bridges between training and faculty positions and insufficient mentoring for research itself, not to mention research that crosses disciplinary boundaries.

Despite these promising opportunities, the extent to which pedagogical approaches reinforce interdisciplinary interaction and problem solving needs to be examined across the spectrum of education, from undergraduate to postgraduate training and continuing education. Problem-based or case-study methods promote models that integrate the disciplines and facilitate broad-based discussions among students in professional schools across Canada. Applying similar methods to graduate research training may also hold some promise.

In the health professions, however, there is ongoing and competing demand for curricular content; new approaches that involve interdisciplinary activities may be regarded as a "luxury." McCarthy ${ }^{20}$ acknowledges that most in academia and industry appreciate the value of individuals with interdisciplinary training but do not know at what stage interdisciplinary training should start. Are innovative postdoctoral fellowships coming too late in a researcher's training, to make a real difference? Could undergraduates be exposed to joint courses or external mentors while they remain in their disciplinary degree program? Should an IDHR culture be built-in from the start, beginning with an overhaul of the graduate admissions process, which currently privileges students based on academic grades in standard curricula? These are just some of the curricular questions to consider, in the attempt to build a culture for IDHR.

It has been said that singular value placed on disciplinary scientific knowledge produces students that lack a broad base of knowledge and skills. ${ }^{21}$ As noted by Ares, ${ }^{22}$ the focus on a "major" in undergraduate education "begins the process of disciplinary allegiance." He suggests that a shift occur so that the declaration of a major can be viewed as exploration of an area of interest rather than as a lifelong commitment to a specific discipline. In recent years, the number of interdisciplinary undergraduate programs has increased in Canada. If any gains from this shift are to be maintained, the structure of postgraduate and research training and academic career advancement must follow suit.

\section{Discussion: How can Canadian academia better accommodate and embrace IDHR?}

After at least 2 decades of experience in building interdisciplinary initiatives to complement strong and solid disciplines in many of Canada's universities, it is an opportune moment for us to comment on what seems to be working and where the continuing challenges lie. We therefore offer some brief observations on the measures that stand to make the most immediate difference in strengthening IDHR in Canadian universities, but also note that the members of the assessment committee are all university-based and cannot really speak for industry and government. These preliminary recommendations can be roughly grouped as follows: resourcing, recognizing and rewarding, and training for IDHR.

By now it is clear that IDHR requires a considerable investment in researcher time, physical space and financial resources. Adequately resourcing IDHR is the combined responsibility of universities and granting agencies. Sufficient funding is needed to allow for the regular and frequent research-team meetings so integral to the success of IDHR projects. ${ }^{23}$ The support of institutional administrators to meet the need for time, workload adjustment and space for researchers who conduct IDHR is vital. In addition, novel administrative and research supports are helpful. These could include positions such as team coordinators, and librarians with expert search skills across disciplinary databases. ${ }^{24}$

With respect to granting agencies, much progress has already been made in IDHR support. CIHR, among others, is beginning to offer innovative requests for proposals that take into account the exigencies of team-based interdisciplinary work, including the need for early seed money. To complement this progress and to promote intersectoral collaboration, research funding agencies could collaborate with industry to create industrial research studentships at all levels of training. Universities could also explore with funding agencies ways in which university-based researchers can receive credit for research dollars in non-investigator-initiated projects, such as partnerships with industry or government.

In addition to considerations for resourcing IDHR, the process by which institutions and professional organizations assess and recognize IDHR is often inadequate or even counterproductive. The peer-review process for IDHR researchers and programs alike should include researchers with expertise in IDHR methodologies. Interdisciplinary research can be assessed by criteria such as the number of publications generated that involve more than 1 discipline. In fact, the Common CV Network process (www.commoncv.net) being developed under the auspices of the National Alliance of Provincial Health Research Organizations (NAPHRO) provides a perfect opportunity to include such criteria.

To hiring and promotion committees, candidates should provide a clear statement of their contributions to all publications and presentations, including statements from coinvestigators on peer-reviewed grants that document their individual input. Similarly, letters from postdoctoral supervisors should clearly identify disciplinary strengths and benefits, not 
only to a department but also to the wider university, with respect to the potential to complement and engage with other programs. Other indicators to consider are candidates' involvement in IDHR training activities, their awareness and understanding of the knowledge and practices from disciplines other than their own, and their readiness to engage in research that crosses these boundaries..$^{25}$ There is a need to develop quantifiable criteria to determine IDHR success; but, meanwhile, institutions should provide incentives for hiring proven team players.

Further incentives to engage in IDHR could include yearly financial awards for merit that are unrelated to base salary, as well as guidelines which clearly indicate that interdisciplinary research will receive special consideration for these awards. Universities could also create specific prizes and grants to reward those working in interdisciplinary research. An example is UBC's Peter Wall Institute for Advanced Studies, which funds interdisciplinary workshops, interdisciplinary grants and a resident scholar program.

Finally, if academia is serious about supporting IDHR, it is important to begin with the most fundamental academic activity: teaching, including research training. In the health sciences specifically, models of interprofessional education should be promoted and rigorously examined as a way to enhance communication and sharing of research cultures and perspectives across professions. Training and education should incorporate pedagogical strategies that promote interdisciplinary problem-solving and the development of group processes and collaboration skills from the undergraduate through the postdoctoral levels. For faculty already engaged in research, universities should develop short-term professional development courses that focus on the skills and interactions that facilitate IDHR. These could be offered in collaboration with major research-funding agencies. There is also a need for assessment, if academia is to meet the needs of industry and government for interdisciplinary researchers. Those needs should be defined by those sectors, then addressed in training programs and work-study experiences that strengthen novel training schemes such as clinicianscientist training programs and the provision of meaningful studentship and postdoctoral support in collaboration with government or industry.

All of these recommendations and initiatives would be wasted, however, if professional colleges and societies do not take steps to recognize and encourage IDHR within the professions. Currently, governance of curriculum and licensing maintain strict disciplinary boundaries through the professional colleges, and professional societies actively discourage publication in anything but discipline-bound journals. These traditions may help create the solid disciplinary foundation so important to the professions, but they effectively prevent meaningful collaboration among disciplines. (Ironically, it is this strong disciplinary foundation that allows for the most fruitful IDHR to take place.)

Canada is internationally renowned for innovation, cooperation and consensus, characteristics that position our country well to show leadership in the adoption of measures that foster the pursuit of IDHR. Canadians are already leaders in the use of information technology to meet the challenges posed by our vast geography, a feat that facilitates national and international research collaborations. Canada is also credited for its leading role in advancing the theory and practice of health promotion. The Ottawa Charter ${ }^{26}$ is widely regarded as one of the most influential statements on health, for its inclusion of the broader social determinants. It has profoundly affected the approach of health care practitioners as well as researchers, not to mention the impact it has had on the spirit and intent of many public granting agencies. Canada therefore is undoubtedly well positioned to be a global leader in interdisciplinary and translational research in the health sciences. The question is, are our universities ready to get us there?

\section{Conclusion: Where do we go from here?}

The intention of this article has been to provide some reflections on the current culture of health research in Canada and the climate for interdisciplinary initiatives. We have argued that despite laudable efforts to fund and structure IDHR, the culture and processes of most Canadian universities and academic health care institutions maintain the traditional discipline-bound frameworks. This argument is based on our years of combined professional experience conducting and promoting IDHR in Canadian academic settings. What is needed now is a true analysis of the state of IDHR, through systematic and rigorous collection of data on programs and policies across Canada. We therefore conclude our discussion with some thoughts on the direction that such an analysis might take.

First, there needs to be an inventory of academic centres, institutes and consortiums that strive to facilitate IDHR. This inventory should include metrics such as the number of funded projects and collaborating researchers, along with qualitative data such as the disciplines represented and the governance and administrative structures of the entities. Comparative data could show how each model deals with hiring, promotion and awards, as well as the university policies and procedures that either facilitate or impede its operation.

A second branch of the research could examine the impact of professional organizations on health research in general and IDHR in particular. How do colleges and societies promote their members and acknowledge achievement in research? What rules govern the type of research that is recognized and suitable for publication?

Third, a systematic review of research training opportunities would be very useful in identification (and eventually replication) of models that work. Who is doing what to train new researchers in IDHR? What programs exist, and at what level of training are they aimed? If the general focus is on postdoctoral/postlicensure research trainees, is the training meeting its interdisciplinary goals? What is the optimal career stage to introduce interdisciplinary thinking and practice in research? What are the typical research needs, and how can these be met by IDHR teams? Will there be a critical shortage of researchers skilled and experienced in IDHR? Such needs assessments will help to develop a plan of action for universi- 
ties that train and support these researchers, and for agencies that fund them.

These are just some of the questions that should be explored through systematic research on models of IDHR across Canada. It is anticipated that related research questions will be generated as the project evolves.

The assessment was reviewed by all members of the panel on multiple occasions, then by the Canadian Institute of Academic Medicine Executive, and finally by all members of the Canadian Academy of Health Sciences Council. Corrections and suggestions from all these groups were incorporated.

From the Divisions of Pediatrics and Medical Genetics (Hall), Faculty of Medicine (Buchan), College of Health Disciplines (Bainbridge) and School of Nursing (Ratner), University of British Columbia, Vancouver, BC; Faculty of Veterinary Medicine (Cribb), University of Calgary, Calgary, and Health Sciences Council (Drummond), University of Alberta, Edmonton, Alta.; Nova Scotia Agricultural College (Hicks), Truro, NS; Faculty of Nursing (Paterson), University of New Brunswick, Fredericton, NB; Department of Pathobiology (Gyles), University of Guelph, Guelph, the Faculty of Health Sciences, School of Nursing (McWilliam), Communication Sciences and Disorders (SkarakisDoyle), University of Western Ontario, London, and the School of Rehabilitation Science (Solomon), McMaster University, Hamilton, Ont.

Competing interests: None declared.

Contributors: Each of the authors took part in several conference calls and an in-person 2-day meeting concerning the content of the article and assessment. In addition, they each contributed some part of the written assessment and participated actively to summarize the article about the assessment. They have all approved the assessment and the article summarizing it.

Acknowledgements: The authors would like to acknowledge the financial support provided by the Peter Wall Institute for Advanced Studies at the University of British Columbia, as well as the valuable intellectual support of its Acting Director, Diane Newell. Funding was primarily provided by the Canadian Institute of Academic Medicine. Kristiann Allen consulted on manuscript preparation.

\section{REFERENCES}

I. Popper KR. Conjectures and refutations: the growth of scientific knowledge. New York: Routledge and Kegan Paul; I963. p. 88

2. Committee on Facilitating Interdisciplinary Research; National Academy of Sciences, National Academy of Engineering, Institute of Medicine. Facilitating interdisciplinary research. Washington: The National Academies Press; 2004.

3. Marts SA. Interdisciplinary research is key to understanding sex differences: report from the Society for Women's Health Research meeting on understanding the biology of sex differences. J Womens Health 2002;1I:50I-9.
4. Stokols D, Harvey R, Gress J, et al. In vivo studies of transdisciplinary scientific collaboration: lessons learned and implications for active living research. Am J Prev Med 2005;28:202-I3.

5. Rosenfield PL. The potential of transdisciplinary research for sustaining and extending linkages between the health and social sciences. Soc Sci Med I992;35:1343-57.

6. Rustgi AK. Translational research: What is it? Gastroenterology I999;II6:I285.

7. Phillipson E, Silverman M. Clinical research in a CIHR world. Clin Invest Med $2002 ; 25: 26-46$

8. Eric Morin; Health Canada. Rock introduces legislation to create the Canadian Institutes of Health Research [press release]. Ottawa: Health Canada; Ig99 Nov 4. Available: www.hc-sc.gc.ca/ahc-asc/media/nr-cp/I999/I999_I3o_e.html (accessed 2006 Jul 26).

9. Clark PG. Survey: health-care finance. The health of nations. Economist 2004;Jul I5:3-I2.

Io. Birnbaum $\mathrm{PH}$. Contingencies for interdisciplinary research: matching research questions with research organizations. Management Science I981;27:1279-93.

II. Giacomini M. Interdisciplinarity in health services research: dreams and nightmares, maladies and remedies. J Health Serv Res Policy 2004;9:177-83.

I2. Kuhn TS. The structure of scientific revolutions. Chicago: The University of Chicago Press; 1970.

I3. Palmer CL. Structures and strategies of interdisciplinary science. J Am Soc Inf Sci 1999;50:242-53

I4. Magill-Evans J, Hodge M, Darrah J. Establishing a transdisciplinary research team in academia. J Allied Health 2002;31:222-6.

I5. Frost SH, Jean PM. Bridging the disciplines: interdisciplinary discourse and faculty scholarship. J Higher Educ 2003;74:119-49. Available: www.provost.wisc.edu /interdisciplinarity/pdf/bridging_disciplines.pdf (accessed 2006 Jul 27).

I6. Richardson B, Cooper N. Developing a virtual interdisciplinary research community in higher education. J Interprof Care 2003;17:173-82.

I7. Golde CM, Gallagher HA. The challenges of conducting interdisciplinary research in traditional doctoral programs. Ecosystems I999;2:28I-5. Available: http://chris .golde.org/filecabinet/ecosystem.pdf (accessed 2006 Jul 27).

I8. Cooper H, Carlisle C, Gibbs T, et al. Developing an evidence base for interdisciplinary learning: a systematic review. J Adv Nurs 200I;35:228-37.

I9. Freeth D, Hammick M, Koppel I, et al. A critical review of evaluations of interprofessional education. London: UK Centre for the Advancement of Interprofessional Education; 2002. Available: www.health.ltsn.ac.uk/publications/occasionalpaper /occasionalpaper02.pdf (accessed 2006 Jul 26).

20. McCarthy J. Tackling the challenges of interdisciplinary bioscience. Nat Rev Mol Cell Biol 2004;5:933-7.

2I. Wear D, Castellani B. The development of professionalism: curriculum matters. Acad Med 2000;75:602-II.

22. Ares M. Interdisciplinary research and the undergraduate biology student. Nat Struct Mol Biol 2004; II:II70-2.

23. Treloar C, Graham ID. Multidisciplinary cross-national studies: a commentary on issues of collaboration, analysis and publication. Qual Health Res 2003;13:924-32.

24. Bates MJ. Learning about the information seeking of interdisciplinary scholars and students. Libr Trends 1996;45:155-64.

25. Aagaard-Hansen J, Ouma JH. Managing interdisciplinary health research - theoretical and practical aspects. Int J Health Plann Manage 2002;17:195-212.

26. World Health Organization. Ottawa Charter for Health Promotion. Available: www .who.int/hpr/NPH/docs/ottawa_charter_hp.pdf (accessed 2006 Jul 26).

Correspondence to: Dr. Judith Hall, BC's Children's Hospital, Department of Pediatrics, 4480 Oak St., Rm. L408, Vancouver BCV6H 3V4; fax 604 875-2530; jhall@cw.bc.ca

\section{Holiday Review 2006 Call for submissions}

Hilarity and good humour ... help enormously in both the study and the practice of medicine ... [I]t is an unpardonable sin to go about among patients with a long face.

— William Osler

Yes, that's right, it's time to send us your creative contributions for CMAJ's Holiday Review 2006. We're looking for humour, spoofs, personal reflections, history of medicine, off-beat scientific explorations and postcards from the edge of medicine.

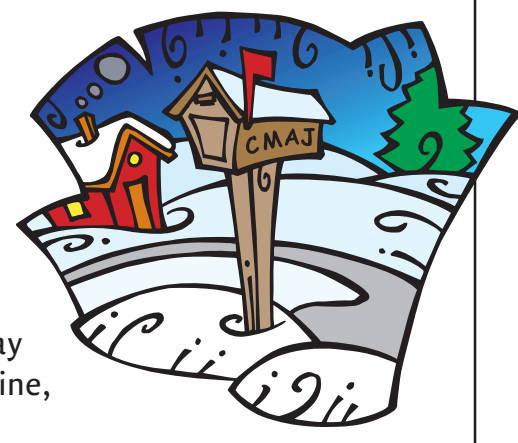

Send your offerings through our online manuscript tracking system (http://mc.manuscriptcentral.com/cmaj). Articles should be no more than 1200 words; photographs and illustrations are welcome. Please mention in your cover letter that your submission is intended for this year's Holiday Review.

The deadline for submissions is Oct. 16, 2006. 